\title{
Tratamento de Hemofílicos A Grave Atendidos no Hospital de Hematologia da Fundação HEMOPE, com Inibidor de Fator VIII Através da Imunotolerância
}

\section{Treatment of Severe Hemophiliacs A Treated at the Hospital de Hematologia da Fundação HEMOPE, with Factor VIII Inhibitor through Immunotolerance} \author{
Magaly do Bom Parto Lopes Vieira Lima ${ }^{\mathrm{b}}$; Antônio Sérgio Alves de Almeida Junior ${ }^{\mathrm{c}}$ \\ a Universidade Federal de Pernambuco. PE, Brasil. \\ ${ }^{b}$ Fundação de Hematologia e Hemoterapia de Pernambuco. PE, Brasil. \\ ${ }^{\mathrm{c} C e n t r o ~ U n i v e r s i t a ́ r i o ~ M a u r i ́ c i o ~ d e ~ N a s s a u . ~ P E, ~ B r a s i l . ~}$ \\ *E-mail: robson.raion@gmail.com
}

Amanda de Oliveira Marinho; ; Abdênego Rodrigues da Silva ${ }^{\mathrm{a}}$; Robson Raion de Vasconcelos Alves;

\begin{abstract}
Resumo
No Brasil, mais de $80 \%$ dos casos de hemofilia são do tipo A. A infusão do concentrado do Fator VIII (FVIII) pode desenvolver anticorpos neutralizantes. $\mathrm{O}$ tratamento de escolha para indivíduos que desenvolveram inibidor é a imunotolerância (IT). Este estudo objetivou avaliar a conduta e eficácia do tratamento da IT em pacientes com hemofilia A grave na Fundação de Hematologia e Hemoterapia de Pernambuco (HEMOPE), no período de 2012 a 2017. O estudo foi do tipo descritivo com uma abordagem quantitativa com análise dos prontuários de pacientes com hemofilia A grave. No total de 17 pacientes hemofílicos A grave, 47\% ainda continuam na IT, 17,7\% tiveram insucesso, 17,7\% foram excluídos do tratamento, $11,7 \%$ conseguiram sucesso parcial e 5,9\% obtiveram sucesso total. A média de idade de detecção do inibidor foi de 4 anos a partir do início do tratamento de infusões, variando de 1 a 14 anos; 7 indivíduos apresentaram o primeiro título de baixa resposta $(<5 \mathrm{UB} / \mathrm{mL})$ e os demais título de alta resposta $(>5 \mathrm{UB} / \mathrm{mL})$; cerca de 70,58\% conseguiram começar a IT com um período de deteç̧ão $<$ 5 anos. A falta de uma conduta padrão diante do tratamento resulta em uma eficácia não definida, apesar de todas as limitações do presente estudo já relatadas, um pequeno percentual de pacientes obteve sucesso parcial e total do tratamento, sendo um indicativo de que a IT realizada, atualmente, nos pacientes hemofílicos A grave da Fundação HEMOPE (iniciada há apenas 6 anos) apresenta resultados promissores.
\end{abstract}

Palavras-chave: Fator VIII. Anticorpo Neutralizante. Eficácia.

\begin{abstract}
In Brazil, more than $80 \%$ of hemophilia cases are type A. Infusion of Factor VIII concentrate (FVIII) can develop neutralizing antibodies. The treatment of choice for individuals who have developed an inhibitor is immunotolerance (IT). This study aimed to evaluate the conduct and effectiveness of treating IT in patients with severe hemophilia A at Fundação de Hematologia e Hemoterapia de Pernambuco (HEMOPE), from 2012 to 2017. The study was the descriptive type with a quantitative approach with analysis of the patients' medical records with severe hemophilia A. In the total of 17 hemophiliac A severe patients, 47\% are still on IT, $17.7 \%$ were unsuccessful, $17.7 \%$ were excluded from treatment, $11.7 \%$ were partially successful and $5.9 \%$ were totally successful. The mean detection age of the inhibitor was 4 years from the beginning of the infusions treatment, ranging from 1 to 14 years; 7 individuals had the first low response titer $(<5$ UB/mL) and the remaining high response titer (> $5 \mathrm{UB} / \mathrm{mL}$ ); about $70.58 \%$ managed to start IT with a detection period $<5$ years. The lack of a standard approach to treatment results in an undefined efficacy, although all the limitations of the present study already reported, a small percentage of patients achieved partial and total treatment success, being an indication that the IT currently performed on severe hemophiliac A patients from Fundação HEMOPE (started only 6 years ago) shows promising results.
\end{abstract}

Keywords: Factor VIII. Neutralizing Antibody. Effectiveness

\section{Introdução}

As hemofilias são doenças hemorrágicas resultantes da deficiência quantitativa ou qualitativa dos fatores de coagulação VIII (hemofilia A) ou IX (hemofilia B), causando assim desequilíbrio no processo de coagulação. Uma mutação genética (geralmente herdada) suprime a produção de proteínas que fazem o sangue coagular. Por ser uma doença recessiva ligada ao cromossomo $\mathrm{X}$ afeta, quase que exclusivamente, os indivíduos do sexo masculino (CARLOS-RIVERA et al., 2016; SANTOS et al., 2018; MUÑOZ et al., 2019).

A hemofilia A da B semelhantes tornando impossível distinguir somente pelos critérios clínicos, sendo necessários exames laboratoriais para o diagnóstico. Os episódios de sangramento ocorrem, principalmente após traumatismos de intensidade mínima, sendo característica da doença os sangramentos musculares (hematomas) e intra-articulares (hematroses), afetando na maioria dos casos as articulações dos joelhos, tornozelos e cotovelos (OSOOLI; BERNTORP, 2015; SANTOS et al., 2019). Há casos mais graves, nos quais os sangramentos podem acometer outros locais do corpo, causando hemorragias internas podendo acometer o sistema nervoso central. Alterações nos fatores de coagulação (VIII e IX) podem desencadear a hemofílicos artropatia baseada em mudanças na função e estrutura articular causadas por processos inflamatórios da sinóvia, bem como pela desintegração da cartilagem (OLDENBURG, 2015; BRASIL, 2017; VAN-VULPEN et al., 2018). 
De acordo com dados da World Federation of Hemophilia, atualmente, existem 12.983 pacientes com hemofilia A e B cadastrados no Brasil, destes mais de $80 \%$ apresentam hemofilia A, esta é a quarta maior população mundial de pacientes com a doença (BRASIL, 2020).

$\mathrm{Na}$ hemofilia A grave cerca de $70 \%$ apresentam sangramentos de 20-30 vezes por ano, frequentemente espontâneos ou causados por traumas. Cerca de $40 \%$ dos casos de hemofilia A grave é causada pela inversão do íntron $22 \mathrm{em}$ que essa mutação inativa por completo o gene do fator VIII (LAVAUT-SÁNCHEZ et al., 2017; CORRÊA et al., 2019).

O tempo de tromboplastina parcial ativado (TTPA) é o teste de triagem que se apresenta prolongado na hemofilia A. O diagnóstico confirmatório é realizado por meio da dosagem da atividade do fator VIII coagulante (BRASIL, 2017).

O tratamento de pacientes hemofílicos inclui a reposição, por via venosa, do concentrado do fator deficiente para prevenir e evitar eventos hemorrágicos. No caso da hemofilia A há reposição do fator VIII que pode ser recombinante ou plasmático. Um dos principais objetivos do tratamento é a prevenção das hemartroses, pois é a alteração que mais causa morbidade a esses indivíduos (OSOOLI; BERNTORP, 2015; BIANCHIN, 2017).

Uma complicação que pode ocorrer através do tratamento de infusão do fator deficiente nos pacientes com hemofilia A grave é o desenvolvimento de anticorpos neutralizantes da classe IgG contra a proteína do fator VIII, chamados de inibidores do fator VIII. Esses anticorpos resultam em uma resposta imune complexa estando relacionada a fatores de riscos, que incluem a própria proteína do fator VIII e as células do sistema imunológico (BATSULI et al., 2016).

Há também fatores externos que podem desencadear o aparecimento do inibidor como o tipo de concentrado de fator (plasmático ou recombinante) e a idade das primeiras infusões. $\mathrm{O}$ grande risco dessa produção acontece até o $50^{\circ}$ dia de infusão. Os indivíduos acometidos passam a não responder à reposição do fator deficiente, podendo apresentar episódios hemorrágicos de difícil controle (CARVALHO et al., 2016).

O Fator Recombinante VII ativado (FVIIr) ou complexo protrombínico parcialmente ativado $(\mathrm{CPPa})$ conhecido como agente by passing é utilizado em eventos hemorrágicos agudos em que o FVIII não tem resposta diante dos inibidores contra a proteína do FVIII, contudo, ainda há discussões sobre o manejo, pois são produtos caros e não apresentam uma hemostasia eficiente em todos os pacientes que o utilizam (PEPE et al., 2017; GRANOVSKI, 2018; SILVA et al., 2019).

Atualmente, o tratamento de escolha para indivíduos que desenvolveram inibidor é a imunotolerância (IT), a única conduta padrão para a reversão clínica (BRASIL, 2017; MONTALVÃO et al., 2019). Consiste na administração intravenosa de doses elevadas de fator VIII diárias ou alternadas durante meses a anos apresentando sucesso em cerca $60-70 \%$ dos indivíduos com anticorpos (BATSULI et al., 2016). Vários critérios são considerados para o sucesso deste tratamento que incluem o começo da IT em idade jovem, título do inibidor inferior ao pico histórico $(<200 \mathrm{UB} / \mathrm{ml})$, título inferior a $10 \mathrm{UB} / \mathrm{mL}$ antes do início da IT, pequenas deleções, inserções e mutações (ANTUN et al., 2015).

A avaliação da terapia de indução a imunotolerância contribuirá para traçar um perfil de tratamento na população hemofílica A grave e, consequentemente, aprimorá-la para aumentar ainda mais a chance desses pacientes terem uma vida consideravelmente normal com a erradicação dos anticorpos contra o fator VIII exógeno.

O objetivo desse estudo é avaliar a conduta e eficácia do tratamento da imunotolerância em pacientes com hemofilia A grave na Fundação de Hematologia e Hemoterapia de Pernambuco (HEMOPE), no período de 2012 a 2017.

\section{Material e Métodos}

Tratou-se de um estudo descritivo com uma abordagem quantitativa realizada no Hospital da Fundação de Hematologia e Hemoterapia de Pernambuco, localizado na cidade do Recife, Pernambuco. Uma instituição que presta serviço assistencial, educacional e científico, estando vinculada à Secretaria de Saúde do Governo de Pernambuco, com atuação nos segmentos de hemoterapia e hematologia. Além de desenvolver ações de saúde na área do sangue, produzindo bens e serviços em diagnóstico laboratorial e tratamento das patologias sanguíneas, atua no desenvolvimento da medicina transfusional e nos serviços de doação de órgãos no Estado.

Os dados utilizados foram extraídos de prontuários físicos de pacientes hemofílicos A grave que finalizaram, permanecem em tratamento ou foram afastados da imunotolerância (IT) por algum motivo no referido hospital, nos anos de 2012 a 2017. Foram excluídos os indivíduos que participaram ou estão participando da IT, porém são hemofílicos A de classificação moderada.

Os parâmetros utilizados nos prontuários foram: idade ao diagnóstico da hemofilia $\mathrm{A}$, a faixa etária de quando apresentou o inibidor e sua titulação, intervalo de tempo entre detecção de inibidor e início da IT, título do inibidor no início da IT, tipo de fator utilizado durante o tratamento, esquema de infusões, duração da IT e quantidade de sangramentos antes e durante a terapêutica e possíveis resultados alcançados.

Com isso, os dados extraídos foram tabulados por meio do programa Microsoft Excel ${ }^{\circledR} 2010$ e submetidos a uma análise estatística descritiva (frequência, proporção e média).

A pesquisa foi realizada de acordo com resolução 466/12 do Conselho Nacional de Saúde, assegurando confidencialidade dos dados e o compromisso ético. O estudo foi aprovado pelo Comitê de Ética em Pesquisa em Seres Humanos da Fundação HEMOPE (CAAE: 84549518.0.0000.5195).

\section{Resultados e Discussão}

O surgimento dos inibidores contra o FVIII é a 
complicação mais grave relacionada ao tratamento da hemofilia A grave. Em episódios hemorrágicos se torna difícil o controle do sangramento, pois o indivíduo não responde a infusão do fator FVIII; aumentando o risco de sangramentos incontroláveis e morbidade, diminuindo a qualidade de vida desses indivíduos (RYU et al., 2015; VALENTINO et al., 2015). No presente estudo, foram avaliados 17 pacientes clinicamente diagnosticados com hemofilia A grave, que apresentaram inibidores contra o fator VIII recebido durante a reposição.

A média de idade ao diagnóstico da hemofilia A grave dos incluídos no estudo foi de 9 meses com intervalo de 1 a 24 meses (Quadro 1). A terapia de substituição é iniciada antes ou após o primeiro evento hemorrágico, sendo administrada o FVIII uma ou duas vezes na semana com o objetivo de manter as concentrações do fator deficiente acima de 1,0 UI/dL, dessa forma evita hemorragias e limita complicações como a artropatia (BENSON et al., 2012; BIMANIS et al., 2012). Segundo Kruse-Jarres (2011), a exposição ao FVIII durante o primeiro ano de vida é uma variável forte para o surgimento de inibidores contra o FVIII.

Quadro 1 - Dados demográficos e características clínicas dos hemofílicos em imunotolerância no HEMOPE, 2012 a 2017

\begin{tabular}{|c|c|c|c|c|c|c|c|c|c|c|}
\hline Indivíduo & $\begin{array}{c}\text { Idade ao } \\
\text { diagnóstico } \\
\text { da } \\
\text { hemofilia } \\
\text { (meses) }\end{array}$ & $\begin{array}{c}\text { Idade de } \\
\text { detecção } \\
\text { do } \\
\text { inibidor } \\
\text { (anos) }\end{array}$ & $\begin{array}{c}\text { Primeiro } \\
\text { título de } \\
\text { inibidor } \\
(\mathrm{UB} / \mathrm{mL})\end{array}$ & $\begin{array}{c}\text { Pico } \\
\text { histórico } \\
\text { (UB/mL) }\end{array}$ & \begin{tabular}{|c|} 
Intervalo \\
entre a \\
detecção \\
do \\
inibidor e \\
IT (meses)
\end{tabular} & $\begin{array}{c}\text { Título do } \\
\text { inibidor } \\
\text { inicial da } \\
\text { IT (UB/ } \\
\text { mL) }\end{array}$ & $\begin{array}{c}\text { Tipo de } \\
\text { FVIII } \\
\text { durante } \\
\text { a IT }\end{array}$ & \begin{tabular}{|c} 
Esquema \\
de infusões \\
(UI/kg/ \\
dose) \\
Frequência
\end{tabular} & $\begin{array}{c}\text { Duração } \\
\text { da IT } \\
\text { (meses) }\end{array}$ & Resultados \\
\hline 1 & 1 & 1 & 0,8 & 27,2 & 8 & 2,4 & FVIII R & $750-3 x$ & 24 & EIT \\
\hline 2 & 11 & 2 & 17,6 & 51,6 & 7 & 11,2 & FVIII R & $100-d^{* *}$ & 12 & EIT \\
\hline 3 & 10 & 12 & 5,5 & 37,2 & 184 & 1 & FVIII R & $50-3 x$ & 33 & EIT \\
\hline 4 & 4 & 2 & 3,2 & 10,4 & 7 & 3,2 & FVIII R & $80-3 x$ & 13 & EIT \\
\hline 5 & 6 & 1 & 5,6 & 19,2 & 12 & 1,6 & FVIII R & $50-3 x$ & 20 & EIT \\
\hline 6 & 10 & 14 & 20 & 179,2 & 144 & 76,8 & FVIII P & $50-3 x$ & 5 & EIT \\
\hline 7 & 1 & 3 & 5,6 & 57 & 48 & 2,6 & FVIII R & $200-d^{* *}$ & 40 & EIT \\
\hline 8 & 5 & 7 & 2,3 & 12,8 & 180 & 1,8 & FVIII P & $50-3 x$ & 13 & EIT \\
\hline 9 & 2 & 2 & 19,2 & 1024 & 60 & 20,8 & FVIII R & - & 36 & INS \\
\hline 10 & 5 & 1 & 1,4 & 116 & 15 & 10,4 & FVIII P* & $750-3 x$ & 18 & EXC \\
\hline 11 & 9 & 1 & 76,8 & 57,6 & 10 & 5,2 & FVIII P* & $200-d$ & 34 & INS \\
\hline 12 & 12 & 5 & 4,4 & 22,4 & 72 & 8 & FVIII P & $100-d$ & 33 & EXC \\
\hline 13 & 24 & 3 & 102 & 115 & 21 & 25,6 & FVIII R & $100-3 x$ & 24 & EXC \\
\hline 14 & 8 & 3 & 1,3 & 12 & 20 & 1,3 & FVIII P* & $200-d$ & 49 & INS \\
\hline 15 & 12 & 3 & 20,8 & - & 48 & 1,8 & FVIII P & $100-3 x$ & 11 & SP \\
\hline 16 & 12 & 2 & 12,8 & 16 & 5 & 5,2 & FVIII R & $71-3 x$ & 17 & SP \\
\hline 17 & 24 & 7 & 3 & 19,2 & 156 & 1,8 & FVIII P & $50-3 x$ & 15 & ST \\
\hline $\begin{array}{l}\text { Média } \pm \\
\text { DP }\end{array}$ & $9,18 \pm 6,54$ & $\begin{array}{c}4,06 \pm \\
3,75 \\
\end{array}$ & $\begin{array}{c}17,78 \pm \\
27,38 \\
\end{array}$ & \begin{tabular}{|c|}
$111,05 \pm$ \\
240,22 \\
\end{tabular} & $\begin{array}{c}58,65 \pm \\
63,13 \\
\end{array}$ & $\begin{array}{c}10,63 \pm \\
17,91 \\
\end{array}$ & - & - & $\begin{array}{c}23,35 \pm \\
17,91 \\
\end{array}$ & - \\
\hline
\end{tabular}

Imunotolerância (IT); Fator VIII recombinante (FVIII-R); Fator VIII plasmático (FVIII-P); Emimunotolerância (EIT); Insucesso (INS); Excluído (EXC); Sucesso Parcial (SP); Sucesso Total (ST);

Diariamente (d); Três vezes semanalmente (3x). * Mudança no esquema de Fator VIII recombinante para Fator VIII plasmático.** Número de infusões aumentou de 3 vezes por semana para todos os dia.

Fonte: Dados da Pesquisa.

Os fatores genéticos do indivíduo desempenham um papel importante por ser um grande preditor do surgimento de inibidores de FVIII (OSOOLI; BERNTORP, 2015). Pacientes com grandes deleções e mutações de alto risco, como as inversões dos íntrons 1 e 22, são mais susceptíveis ao desenvolvimento de inibidores (PREZOTTI, 2016). Os polimorfismos em genes imunorregulatórios associados com a função imunológica (IL-10, TNF- $\alpha$ e CTLA-4) são associados a uma predisposição para o surgimento dos inibidores contra o FVIII (PREZOTTI, 2016); porém os indivíduos do estudo não apresentaram a genotipagem.

A média de idade de deteç̧ão do inibidor foi de 4,0 anos a partir do início do tratamento de infusões, com desvio padrão de $\pm 3,75$ (Quadro 1). Os indivíduos 1, 2, 4, 5, 9, 10,
11 e $16(47,05 \%)$ que apresentaram inibidores entre 1 a 2 anos de idade demonstraram uma associação com os relatos de DiMichele (2004) e Chalmers et al. (2007) nos quais o desenvolvimento de inibidores acontece com média de idade entre os dois primeiros anos de vida. A quantidade de exposições ao FVIII até o desenvolvimento de inibidores não foi aplicado à pesquisa, no entanto, os indivíduos 3, 6, 7, 8, $12,13,14,15$ e $17(52,94 \%)$, que tinham idades entre 3 a 14 anos se relacionaram com o estudo feito por Kempton et al. (2006), que mostrava uma prevalência de inibidores em idade de 2 a 14 anos.

O primeiro título apresentado de inibidor obteve média de $17,7 \mathrm{UB} / \mathrm{mL}$, desvio padrão de $\pm 27,38 \mathrm{em}$ que os indivíduos $1,4,8,10,12,14$ e $17(41,18 \%)$ apresentaram um título de 
baixa resposta $(<5 \mathrm{UB} / \mathrm{mL})$ os demais $2,3,5,6,7,9,11$, 13,15 e $16(58,82 \%)$ títulos de alta resposta $(>5 \mathrm{UB} / \mathrm{mL})$ (Quadro 1). O período entre detecção do inibidor e início da IT com média de 59 meses e intervalo de 5 a 184 meses; os indivíduos 3, 6, 8, 12 e 17 (29,41\%) começaram a IT após um período de detecção do inibidor $>5$ anos e os indivíduos 1,2 , $4,5,7,9,10,11,13,14,15$ e $16(70,58 \%)$ iniciaram em um período $<5$ anos.

É considerado um bom preditor de sucesso, quando o início da IT é $<5$ anos. Em um estudo de indução a imunotolerância e análise dos preditores de sucesso, relatado por Mariani et al. (2001), foram analisados dados retrospectivos de 314 pacientes, mostrando que os mesmos começaram a IT com menos de cinco anos de detecção sendo obtida uma taxa de sucesso superior a $70 \%$.

Comparando o primeiro título apresentado e o título inicial da IT, cerca de $70,5 \%$ dos indivíduos conseguiram iniciar com título $<10 \mathrm{UB} / \mathrm{mL}$, considerado por muitos estudos como mais um preditor de sucesso ao tratamento. Foi impossível analisar essa variável sozinha, pois essa sempre é analisada com os demais preditores. Foi visto que os pacientes 2, 6 , 9, 10 e 13(29,41\%) começaram a IT com o título inicial do inibidor $>10 \mathrm{UB} / \mathrm{mL}$, porém os indivíduos 2 e 6 permanecem em IT, o 9 considerado insucesso e o 10 e 13 excluídos do tratamento (Quadro 1).

Os pacientes analisados tinham um pico histórico com média de 111,05 UB/mL, (min 10,4 UB/mL; máx $1024 \mathrm{UB} /$ $\mathrm{mL}$ ). O pico histórico $<200 \mathrm{UB} / \mathrm{mL}$ é considerado por muitos estudos de coorte como um bom preditor para o sucesso da IT (VALENTINO et al., 2015). O pico histórico é referido como o maior título de inibidor apresentado pelo indivíduo antes do tratamento da IT. Os resultados evidenciam que apenas o indivíduo 9 apresentou um pico histórico acima de $200 \mathrm{UB} /$ $\mathrm{mL}$, resultando em uma média e desvio padrão altos, quando calculado junto dos demais indivíduos, esse valor de pico histórico do indivíduo 9 é considerado um outlier, podendo trazer prejuízo a variável analisada. Porém o mesmo estava no grupo de insucesso à IT, pois ao completar 36 meses de tratamento o inibidor continuava com título elevado.

Por apresentar $47 \%$ dos indivíduos ainda em tratamento, não poderia haver um resultado concreto quando o pico histórico é um dos critérios para obtenção de um bom resultado. Contudo, o indivíduo 17, que obteve o sucesso total ao tratamento, tinha um pico histórico $<200 \mathrm{UB} / \mathrm{mL}$.

$\mathrm{Na}$ pesquisa $52,9 \%$ dos indivíduos faziam a IT com o fator VIII recombinante. Em um estudo demonstrado por Goudemand et al. (2006) em 148 hemofílicos A, foi feita uma comparação entre os tipos de fatores utilizados, 14 indivíduos desenvolveram inibidores contra o FVIII após a utilização do FVIII derivado de plasma, no entanto 49 desenvolveram inibidores contra o FVIII recombinante. Há um aumento do risco de 2,5 a 3,0 vezes em relação ao surgimento de inibidores contra o FVIII, quando os pacientes hemofílicos são tratados com o FVIII recombinante comparando-se aos tratados com o
FVIII plasmático.

Em uma avaliação com portadores de hemofiliaA, Carvalho et al. (2016), no Hemocentro do Ceará, avaliaram um total de 184 indivíduos entre eles $19 \%$ apresentaram anticorpos contra o FVIII, após um tratamento com FVIII recombinante. Porém, em conclusão ao estudo foi recomendado que indivíduos que ainda não começaram o tratamento de reposição ao FVIII, não fossem expostos ao FVIII recombinante evitando assim o surgimento de anticorpos contra o mesmo.

Dos indivíduos que apresentaram inibidores, 47\% continuam em IT, $17,7 \%$ não responderam ao tratamento sendo considerados insucesso, $17,7 \%$ foram excluídos por irregularidades durante a terapêutica, $11,7 \%$ conseguiram sucesso parcial e 5,9\% obteve sucesso total da IT (Quadro 1).

Os pacientes 10, 12 e 13 (17,64\%), que foram excluídos da IT, por não conseguirem seguir as recomendações sobre o tratamento, faltando às consultas, não fazendo a infusão do fator nos dias corretos e, assim, não responderam ao tratamento (Quadro 1). O diagnóstico de inibidores em indivíduos com hemofilia A grave pode provocar sentimentos de tristeza, de angústia e de raiva aos pacientes e pais, segundo Benson et al. (2012), o fornecimento de informações dos profissionais da saúde e médicos à família e aos pacientes seria uma alternativa de compreensão sobre adesão correta a IT, aumentando assim, as taxas de sucesso ao tratamento.

Os indivíduos 2 e 7 (11,76\%) tiveram mudança de esquema das doses de infusão, o 2 mudou de 50 UI FVIII recombinante 3 vezes na semana para 100 UI diariamente e o 7 teve a substituição do seu primeiro esquema de 50 UI 3 vezes semanais por 200 UI diariamente (Quadro 1).

Por falta de um tratamento padronizado, os resultados ficaram limitados para definir a IT, um exemplo é o trabalho publicado por Valentino et al. (2015), que demonstra a conduta do International ITI Registry (IITR) que registrou uma amostra de 204 indivíduos com hemofilia A e inibidores que utilizaram três protocolos diferentes de concentrado de FVIII: $<50$ UI/Kg/dia; 50 a $<200 / \mathrm{UI} / \mathrm{Kg} /$ dia e $\geq 200 \mathrm{UI} /$ $\mathrm{Kg} /$ dia, em que cerca de $67,7 \%$ conseguiram a tolerância completa, no mesmo estudo foi demonstrado os dados do North American ITI Registry (NAITR) com 164 enfermos, em que $51 \%$ receberam $\geq 100 \mathrm{UI} / \mathrm{Kg} /$ dia e $14 \%$ receberam doses diárias $\geq 200 \mathrm{UI} / \mathrm{Kg} /$ dia, resultando em uma taxa de sucesso de $70 \%$ entre os envolvidos.

Em uma análise retrospectiva, realizada por Ryu et al. (2015), em hemofílicos A grave com inibidores durante os anos de 2004 a 2014 no Korea Hemophilia Foundation Clinic, os mesmos utilizaram um regime da IT com 3 fases. A primeira fase da indução iniciou com a administração do concentrado de FVIII na dose 100 UI/Kg três vezes semanais com duração de 12 a 48 semanas, durante esse período foi dosado o título do inibidor e FVIII de recuperação, verificando-se a cada duas semanas, no caso de diminuição constante do inibidor, foram administradas doses do concentrado de FVIII diariamente por um período de mais de 48 semanas, após uma nova dosagem 
se os títulos do inibidor persistissem elevados ou uma resposta anamnésica de 10 semanas, os mesmos seriam considerados como falhos e retirados da IT.

Fazendo uma comparação com o protocolo adotado no Brasil com o empregado na Korea Hemophilia Foundation Clinic, o inibidor do indivíduo é dosado a cada consulta realizada no HEMOPE, porém o teste de recuperação do FVIII só é feito ao final do tratamento da IT. O teste de recuperação tem como objetivo expressar a relação da atividade máxima do FVIII no plasma após a infusão do FVIII exógeno.

Segundo o Ministério da Saúde, no Brasil, a indução da IT pode empregar diferentes doses, como o esquema de baixas doses $(50 \mathrm{UI} / \mathrm{Kg} / \mathrm{dia})$ três vezes semanais, que é indicado para início da IT em todos pacientes incluídos ao tratamento e o esquema de doses altas $100 \mathrm{UI} / \mathrm{Kg} /$ diariamente, quando há ausência de declínio do título do inibidor em menos de $20 \%$ desde do início da IT (CARVALHO et al., 2016). Com isso, esta pesquisa evidencia o esquema de infusões do concentrado de FVIII diferente para cada indivíduo, que varia de $50 \mathrm{UI} / \mathrm{Kg}$ três vezes na semana a $200 \mathrm{UI} / \mathrm{Kg}$ diariamente dependendo da resposta de cada paciente.

Os indivíduos 11 e $14(11,76 \%)$ tiveram mudança no tipo de fator VIII utilizado durante o tratamento, obtendo falta de resposta ao fator VIII recombinante, no entanto, já estavam no grupo do insucesso a IT por não ter alcançado êxito ao tratamento no período determinado de 33 meses como recomenda o Ministério da Saúde (Quadro 1). Segundo o protocolo da IT implantado pelo Ministério da Saúde, a troca do tipo de concentrado de FVIII recombinante para plasmático pode ser feita para aqueles, que iniciaram a IT e têm ausência de declínio de pelo menos $20 \%$ do pico máximo do título do inibidor em até 6 meses, a partir do início da IT.

Os pacientes 15 e $16(11,76 \%)$ que alcançaram o sucesso parcial ao tratamento apresentaram um tempo médio de tolerância de 14 meses. Segundo o protocolo da IT implantado pelo Ministério da Saúde em 2011, são levados em consideração quatro parâmetros farmacocinéticos para o resultado final do tratamento: o título de inibidor negativo (< $0,6 \mathrm{UB} / \mathrm{mL}$ ) em duas dosagens com intervalo de 30 dias; teste normal de recuperação do FVIII coagulante acima de 66\%; uma vida média do FVIII $>6$ horas após 72 horas sem infusão de concentrado de fator VIII exógeno e ausência de resposta anamnésica em uma exposição ao fator (Quadro 2).

Quadro 2 - Parâmetros farmacocinéticos finais do tratamento da imunotolerância em pacientes do HEMOPE, 2012 a 2017

\begin{tabular}{|c|c|c|c|c|c|}
\hline \multicolumn{2}{|c|}{$\begin{array}{l}\text { Duas Dosagens com Intervalo de } \\
30 \text { dias }(\mathrm{UB} / \mathrm{mL})\end{array}$} & $\begin{array}{l}\text { Teste de recuperação } \\
\text { FVIII: C * }\end{array}$ & $\underset{* *}{\text { Vida média ao fator }}$ & $\begin{array}{c}\text { Resposta em } \\
\text { reexposição }(\mathrm{UI} / \mathrm{mL})\end{array}$ & Resultados \\
\hline \multirow{2}{*}{15} & $1^{\circ} 0,05$ & \multirow{2}{*}{$35,1 \%$} & \multirow{2}{*}{6,01} & \multirow{2}{*}{0,0} & \multirow{2}{*}{ Sucesso Parcial } \\
\hline & $2^{\circ} 0,0$ & & & & \\
\hline \multirow{2}{*}{16} & $1^{\circ} 0,0$ & \multirow{2}{*}{$66,6 \%$} & \multirow{2}{*}{4,65} & \multirow{2}{*}{0,05} & \multirow{2}{*}{ Sucesso Parcial } \\
\hline & $2^{\circ} 0,05$ & & & & \\
\hline \multirow{2}{*}{17} & $1^{\circ} 0,05$ & \multirow[b]{2}{*}{$71,3 \%$} & \multirow[b]{2}{*}{7,3} & \multirow[b]{2}{*}{0,0} & \multirow{2}{*}{ Sucesso Total } \\
\hline & $2^{\circ} 0,0$ & & & & \\
\hline
\end{tabular}

Unidade Bethesda (UB); Unidade Internacional (UI); *> 66\% = Teste Normal; **> 6 horas $=$ Teste Normal

Fonte: Dados da pesquisa.

O indivíduo $17(5,9 \%)$ que obteve o sucesso total a IT apresentou as duas últimas dosagens com intervalo de 30 dias com valores de $0,05 \mathrm{UB} / \mathrm{mL}$ e $0,0 \mathrm{UB} / \mathrm{mL}$, com teste de recuperação de $71,3 \%$, vida média do fator de 7,3 horas e sem resposta a reexposição do fator (Quadro 2). Por ter um pequeno quantitativo de indivíduos que obtiveram algum resultado da IT, foi impossível fazer comparações com outros estudos e cerca de $47 \%$ ainda estava em IT e não tendo resultado de conclusão de tratamento.

Todos os pacientes incluídos no tratamento possuíam histórico de hemartroses, alguns com artropatia instalada em joelhos, cotovelos ou tornozelos. Eventos hemorrágicos eram constantes antes e durante a IT: hematúria, epistaxe, gengivorragia, hematomas, equimoses muitas vezes espontâneas ou por alguns traumas. Porém, durante a IT a frequência desses eventos foram maiores (Quadro 3).
Quadro 3 - Manifestações clínicas apresentadas antes e durante o tratamento da imunotolerância em pacientes do HEMOPE, 2012 a 2017

\begin{tabular}{|c|c|c|}
\hline \multirow{2}{*}{$\begin{array}{l}\text { Manifestações } \\
\text { Clínicas }\end{array}$} & \multicolumn{2}{|c|}{ Imunotolerância } \\
\hline & Antes da IT & Durante a IT \\
\hline Hermartrose & 5 & 8 \\
\hline Artropatia & 3 & 5 \\
\hline $\begin{array}{c}\text { Eventos } \\
\text { hemorrágicos } *\end{array}$ & 6 & 12 \\
\hline Total & 14 & 25 \\
\hline
\end{tabular}
equimoses

Fonte: dados da pesquisa.

Os indivíduos deste estudo, quando apresentavam eventos hemorrágicos, o FVIIr e CCPa eram utilizados para interromper o sangramento e minimizar os danos causados por esses episódios. É descrito no protocolo de indução 
a IT no Brasil que a escolha do agente bypassing acontece através da resposta do indivíduo ao produto, porém o $\mathrm{CPPa}$ é o que possuí uma boa resposta em sua utilização e, ainda, apresenta uma vida média mais longa comparado ao FVIIIr (CARVALHO et al., 2016).

Os pacientes 10,12 e 13 excluídos e 9, 11, 14, que tiveram insucesso a IT, fazem atualmente profilaxia secundária de longa duração (PSLD) com CPPa em doses que variam de 50 $\mathrm{UI} /$ dose diariamente ou 2 vezes na semana para evitar eventos hemorrágicos agudos após fracasso ao tratamento da IT.

As principais limitações foram o pequeno número de indivíduos e as inerentes a um estudo do tipo descritivo baseado em revisão de prontuários, porém reflete a realidade local.

\section{Conclusão}

O manejo com pacientes hemofílicos A grave com inibidores é um desafio enfrentado por inúmeros centros de especialização hematológica. Várias condutas no tratamento da IT são empregadas para o alcance do sucesso na erradicação dos inibidores contra o FVIII e restabelecer a resposta ao concentrado de fator utilizado para reposição. A falta de uma conduta padrão diante do problema resulta em uma eficácia não definida.

No entanto, apesar de todas as limitações do presente estudo já relatadas, um pequeno percentual de pacientes obteve sucesso parcial e total, o que é um indicativo de que a IT realizada, atualmente, nos pacientes hemofílicos da Fundação HEMOPE (iniciada há apenas 6 anos) apresenta resultados promissores.

\section{Referências}

ANTUN, A. et al. Inhibitor recurrence after immune tolerance induction: a multicenter retrospective cohort study. J. Thromb. Haemostasis, v.13, n.11, p.1980-1988, 2015. doi: 10.1111/ jth. 13143

BATSULI, G. et al. Innovating immune tolerance induction for haemophilia. Haemophilia, v.22, p.31-35, 2016. doi: 10.1111/ hae. 12989

BENSON, G. et al. Immune tolerance induction in patients with severe hemophilia with inhibitors: expert panel views and recommendations for clinical practice. Euro. J. Hematol., v.88, n.5, p.371-379, 2012. doi: 10.1111/j.1600-0609.2012.01754.x

BIANCHIN, P. Atuação do fisioterapêuta nos pacientes hemofilicos com hemartrose. 2017.

BIMANIS, J. et al. Concentración de inhibidores anti VIII en pacientes con hemofilia A que acudieron a una consulta hematológica. Salus, v.16, n.3, p.26-32, 2012.

BRASIL. Ministério da Saúde.Secretaria de Atenção à Saúde. Departamento de Atenção Especializada e Temática. Perfil das coagulopatias hereditárias no Brasil. Brasília: MS, 2017.

BRASIL. HemofiliaLta Brasilia: MS, 2020.

CARLOS-RIVERA, F. et al. Impacto Económico de la Hemofilia tipo A y B en México. Gaceta Med. Mexico, v.152, n.1, p.19-29, 2016.
CARVALHO, R.A. et al. Incidence of factor VIII inhibitory antibodies in patients with hemophilia A seen at HEMOCE, Ceará, Brazil. J. Bras. Patol. Med. Laboratorial, v.52, n.6, p.382386, 2016.doi: 10.5935/1676-2444.20160056

CHALMERS, E.A. et al. Early factor VIII exposure and subsequent inhibitor development in children with severe haemophilia A. Haemophilia, v.13, n.2, p.149-155, 2007. doi: 10.1111/j.1365-2516.2006.01418.x

CORRÊA, M. C.S.M. et al. Prevalência das inversões nos íntrons 1 e 22 do gene do fator VIII e inibidores em pacientes do sul do Brasil. J. Bras. Patol. Med. Laboratorial, v.55, n.6, p.598-605, 2019. doi: $10.5935 / 1676-2444.20190053$

DIMICHELE, D. M. Inhibidores en hemofilia: información básica. Canadá: Federación Mundial de la Hemofilia, 2004.

GOUDEMAND, J. et al. Influence of the type of factor VIII concentrate on the incidence of factor VIII inhibitors in previously untreated patients with severe hemophilia A. Blood, v.107, n.1, p.46-51, 2006. doi: 10.1182/blood-2005-04-1371

GRANOVSKI, V. Purificação de fatores de coagulação VIII e VII recombinantes para o tratamento das hemofilias A e B produzidos a partir de células humanas. São Paulo: Universidade de São Paulo, 2018.

KEMPTON, C. L. et al. Incidence of inhibitors in a cohort of 838 males with hemophilia A previously treated with factor VIII concentrates. J. Thrombosis Haemostasis, v.4, n.12, p.2576-2581, 2006. doi: 10.1111/j.1538-7836.2006.02233.x

KRUSE-JARRES, R. Current controversies in the formation and treatment of alloantibodies to factor VIII in congenital hemophilia A. Hematology 2010. Am. Soc. Hematol. Educ. Prog. Book, v.2011, n.1, p.407-412, 2011. doi: 10.1182/ asheducation-2011.1.407

LAVAUT-SÁNCHEZ, K. Importancia del diagnóstico genético en hemofilia. Rev. Cubana Hematol. Inmunol. Hemoter., v.33, n.S1, 2017.

MARIANI, G. et al. Immune tolerance in hemophilia with factor VIII inhibitors: predictors of success. Haematologica, v. 86, n. 11, p. 1186-1193, 2001. doi: 10.3324/\%25x

MONTALVÃO, S. et al. Cellular and humoral immune response to factor VIII in patients with hemophilia $A$ and inhibitor submitted to the protocol of induction of immune tolerance. 2019.

MUÑOZ, L. et al. Alteraciones en el tromboelastograma de pacientes con hemofilia tipos A y B llevados a cirugía y su correlación con las transfusiones en el Hospital de San José en el periodo de 2010 a 2017. Estudio de tipo corte transversal. Acta Colombiana de Cuidado Intensivo, v. 19, n. 3, p. 125-132, 2019. doi: 10.1016/j.acci.2019.02.002

OLDENBURG, J. Optimal treatment strategies for hemophilia: achievements and limitations of current prophylactic regimens. Blood, J. Am. Soc. Hematol., v.125, n.13, p.2038-2044, 2015. doi: 10.1182/blood-2015-01-528414

OSOOLI, M.; BERNTORP, E. Inhibitors in haemophilia: what have we learned from registries? A systematic review. J. Int. Med., v.277, n. 1, p. 1-15, 2015. doi: 10.1111/joim.12301

PEPE, C. et al. Custo-efetividade do fator VII ativado recombinante (rFVIIa) e complexo protrombínico parcialmente ativado $(\mathrm{CCPa})$ no tratamento sob demanda do sangramento leve/ moderado de pacientes com hemofilia congênita complicada por inibidores sob a perspectiva do sistema público de saúde do Brasil. JBES: Braz. J. Health Econom., v.9, n.3, 2017.

PREZOTTI, A. N. L. et al. Indução da imunotolerância em pacientes adultos com hemofilia A e inibidor. 2016. 
RYU, J. E. et al. Immune tolerance induction in patients with severe hemophilia A with inhibitors. Blood Res., v.50, n.4, p.248$253,2015$.

SANTOS, C. A. et al. Perfil epidemiológico dos hemofílicos atendidos no Centro de Hemoterapia de Sergipe. In: SEMANA DE PESQUISA DA UNIVERSIDADE TIRADENTESSEMPESq, n. 19, 2018.

SANTOS, M. A. et al. Diagnóstico de hemofilia: a necessidade de informações e campanhas educacionais para os familiares de hemofílicos. Olhar de Professor, v.22, p.1-14, 2019. doi: 10.5212/OlharProfr.v.22.0019
SILVA, E. G. S. et al. Tratamento endovascular de pseudoaneurisma da artéria subclávia em criança hemofílica. J. Vascular Bras., v.5, n.2, p.151-156, 2019.

VALENTINO, L.A. et al. US Guidelines for immune tolerance induction in patients with haemophilia a and inhibitors. Haemophilia, v.21, n.5, p.559-567, 2015. doi: 10.1111/ hae. 12730

VAN-VULPEN, LF.D. et al. Joint disease in haemophilia: Pathophysiology, pain and imaging. Haemophilia, v.24, p.44-49, 2018. doi: 10.1111/hae.13449. 
